\title{
Composition, abundance and biomass of zooplankton in Orinoco floodplain lakes, Venezuela
}

\author{
E. Vásquez ${ }^{1}$ \\ J. Rey²
}

Keywords : Zooplankton, species composition, abundance, biomass, floodplain lakes, Orinoco River, Venezuela.

Zooplankton samples were collected over a 21 -month period in five floodplain lakes of the Orinoco River in order to establish zooplankton species composition, abundance patterns, and biomass and their relationships with the patterns of inundations and lake morphometry. 60 rotifer taxa were identified. This group was generally more abundant than copepods and cladocerans (mean : $73,4 \%$ of total zooplankton). Common and abundant rotifer species included $K$. americana, $K$. cochlearis, $B$. mirus, $B$. gessneri, $P$. vulgaris and $F$. longiseta. Copepods were dominated by nauplii (mean : $73,8 \%$ of total copepod stages). Cladocerans were scarce and dominated by $M$. minuta, $C$. cornuta and $D$. spinulosum. Most frequent and abundant zooplankton species were euplanktonic with a dominance of filter feeders and microphagous detritivores. Mean zooplankton density in all lakes ranged from 340 ind. $/ 1$ to 3486 ind./l. Biomass ranged from $71,1 \mu \mathrm{g} .1$ (dw) to $432,8 \mu \mathrm{g} / \mathrm{l}$. Rotifers accounted for $64,7 \%$ of the total mean zooplankton biomass in three lakes while copepods accounted for $57,8 \%$ of the biomass in two lakes. Both density and biomass were markedly seasonal with highest mean values at low waters. Mean density in the lakes was 100 times higher than in the Orinoco main stem. Lakes with highest variabilities in surface area and water depths showed highest zooplankton densities. The type of connection (direct or indirect) established between the lakes and the major source of the water also seemed important to interpret the productivity of floodplain lakes.

\section{Composition, abondance et biomasse du zooplancton dans quelques lacs d'inondation de l'Orénoque (Vénézuela)}

Mots clés : Zooplancton, composition spécifique, abondance, biomasse, lacs d’inondation, Orénoque, Vénézuela.

Des échantillons ont été recueillis durant 21 mois dans 5 lacs de la plaine d'inondation de l'Orénoque afin d'établir la composition spécifique, l'abondance et la biomasse du zooplancton et leurs corrélations avec les caractéristiques de l'inondation et de la morphométrie des lacs. 60 taxa de rotifères ont été identifiés. Ce groupe s'est généralement révélé plus abondant que les copépodes et les cladocères $(73,4 \%$ en moyenne du zooplancton total) avec, comme espèces les plus abondantes et les plus communes : K. americana, $K$. cochlearis, $B$. mirus, $B$. gessneri, $P$. vulgaris et $F$. longiseta. Les copépodes ont été essentiellement représentés par des nauplii $(73,8 \%$ en moyenne du total des stades de développement). Les cladocères, moins abondants, ont été dominés par $M$. minuta, $C$. cornuta et $D$. spinulosum. Les espèces zooplanctoniques les plus fréquentes et les plus abondantes étaient des formes euplanctoniques avec une dominance de filtreurs et de détritivores microphages. Dans tous les lacs, la densité moyenne du zooplancton a varié de 340 ind. $/ 1$ à 3486 ind. /, et la biomasse, de $71,1 \mu \mathrm{g} / \mathrm{l}(\mathrm{dw})$ à $432,8 \mu \mathrm{g} / \mathrm{l}$. Les rotifères ont constitué $64,7 \%$ de la biomasse moyenne du zooplancton dans 3 lacs, les copépodes, $57,8 \%$ de la biomasse dans les 2 autres lacs. Densité et biomasse ont montré les valeurs moyennes les plus hautes à basses eaux. La densité moyenne dans les lacs s'est révélée environ 100 fois plus élevée que dans le canal principal de l'Orénoque. Les plus fortes densités de zooplancton ont été observées dans les lacs offrant les plus grandes variations de surface et de profondeurs d'eaux. Le type de connection (directe ou indirecte) entre les lacs et la source principale des eaux semble être un facteur important pour l'interprétation de la productivité des lacs de la plaine d'inondation.

1. Fundación La Salle de Ciencas Naturales, Estación Hidrobiológica de Guyana, Apartado 51, San Félix, Edo, Bolivar, Venezuela.
2. Laboratoire d'Hydrobiologie, Université Paul Sabatier, UA 695 du CNRS, 118, route de Narbonne, 31062 Toulouse Cédex, France. 


\section{Introduction}

Wetzel (1990) discussed the aspects linked to the role played by land-water interfaces and their high productivity. In his examination of the number of lakes in the world in relation to lake area and mean water depth, he found an overwhelming global predominance of small shallow lakes.

In fluvial floodplain systems, wetlands and littoral components highly contribute to low ratios of pelagic/wetland + littoral. Junk et al. (1989) divide the river floodplain system into permanent lotic habitats, permanent lentic habitats, and the aquatic/terrestrial transition zone.

Despite the fact that floodplain lakes probably best represent lowland tropical lakes (Tundisi et al 1984) and considering their importance in the metabolic processes associated to floodplain river systems, information on this type of waterbodies is rather scarce compared to other more classical lakes.

In South America, floodplain areas have been estimated in $-300000 \mathrm{~km}^{2}$ (Welcomme 1985). On the Orinoco River floodplain $\left(-7000 \mathrm{~km}^{2}\right)$ some 2300 permanent lakes have been identified (Hamilton \& Lewis, 1990a). Morphometric features of some of these lakes were investigated by Vásquez (1988, 1989). These waterbodies show a dynamics in their morphometric characteristics under the influence of the hydrological regime of the river. Physical, chemical, and biological variables in these lakes have been mainly explained by the seasonality of river discharge and basin morphology (Vásquez \& Sánchez 1984, Hamilton \& Lewis 1987 , 1990 b).

Considering that contribution of lake biomass to rivers may be high during inundations (Junk 1984), relatively few studies have been done in Orinoco lakes to evaluate the dynamics and production of their plankton communities. In a large lake, Vásquez and Sánchez (1984) found highest plankton density at low waters. Twombly \& Lewis (1987) observed in a small floodplain lake that total zooplankton population sizes might in fact be larger at high waters due to large changes in lake volumes. Zooplankton densities were also found to be affected by retention of organisms by floating macrophyte beds (Hamilton et al. 1990).

The purpose of this paper is to present information on zooplankton density, seasonality and biomass from a set of five Orinoco floodplain lakes. The research was aimed at establishing the ranges of variations of the previous aspects of zooplankton communities in relation to the seasonality of inundation and lake basin morphology. This is the first long-term study of zooplankton communities developed in several lakes on the Orinoco River floodplain. The results of this study may also prove valuable to improve current classification schemes of freshwater bodies in the world (Higler \& Statzner 1988) mainly based on data from the temperate region.

\section{Research sites}

Zooplankton samples were collected in five permanent floodplain lakes : L. Playa Blanca, L. Orsinera, L. Jobera, L. Lagoven, and L. Río Claro (Fig. 1). Based on Drago's classification of floodplain lakes (Drago 1976), Vásquez $(1988,1989)$ classified them (except L. Río Claro) as lateral levee lakes (type 13). This is a very frequent type of lake in the area of study. L. Rio Claro was classified as a confluence lake (type 11) formed by the regressive inundations of the River Upata (intermittent water course). L. Río Claro shows an indirect connection with the Orinoco River (Connection type 2.3 , in the sense of Drago 1981) while the remaining lakes show a direct connection with the Orinoco River (connection type 1.1). A detailed morphological description of the lakes is given by Vásquez (1989). Table 1 shows some morphometric features of these waterbodies at low and high waters. From the morphometric data for both periods, highest percentage of variation corresponds to water depth followed by surface, perimeter, maximum breadth, and the shore development factor.

In the area of study, the Orinoco River hydrograph usually shows an amplitude ranging from $10 \mathrm{~m}$ to $12 \mathrm{~m}$ (Fig. 2). In a 24 -month period (October 1983 - September 1985), lake water depths ranged from $0,1 \mathrm{~m}$ (L. Río Claro) to $5,8 \mathrm{~m}$ (L. Orsinera) with a general mean value of $1,7 \pm 0,6 \mathrm{~m}$ (Table 1). Based of depth data, Vásquez (1989) distinguished four hydrological phases in these water bodies (Fig. 3) : low water phase (December-June), rising water phase (July-August), high water phase (August-September) and falling water phase (October-November). Figure 4 shows estimates of lake volumes. These were made using the formula 


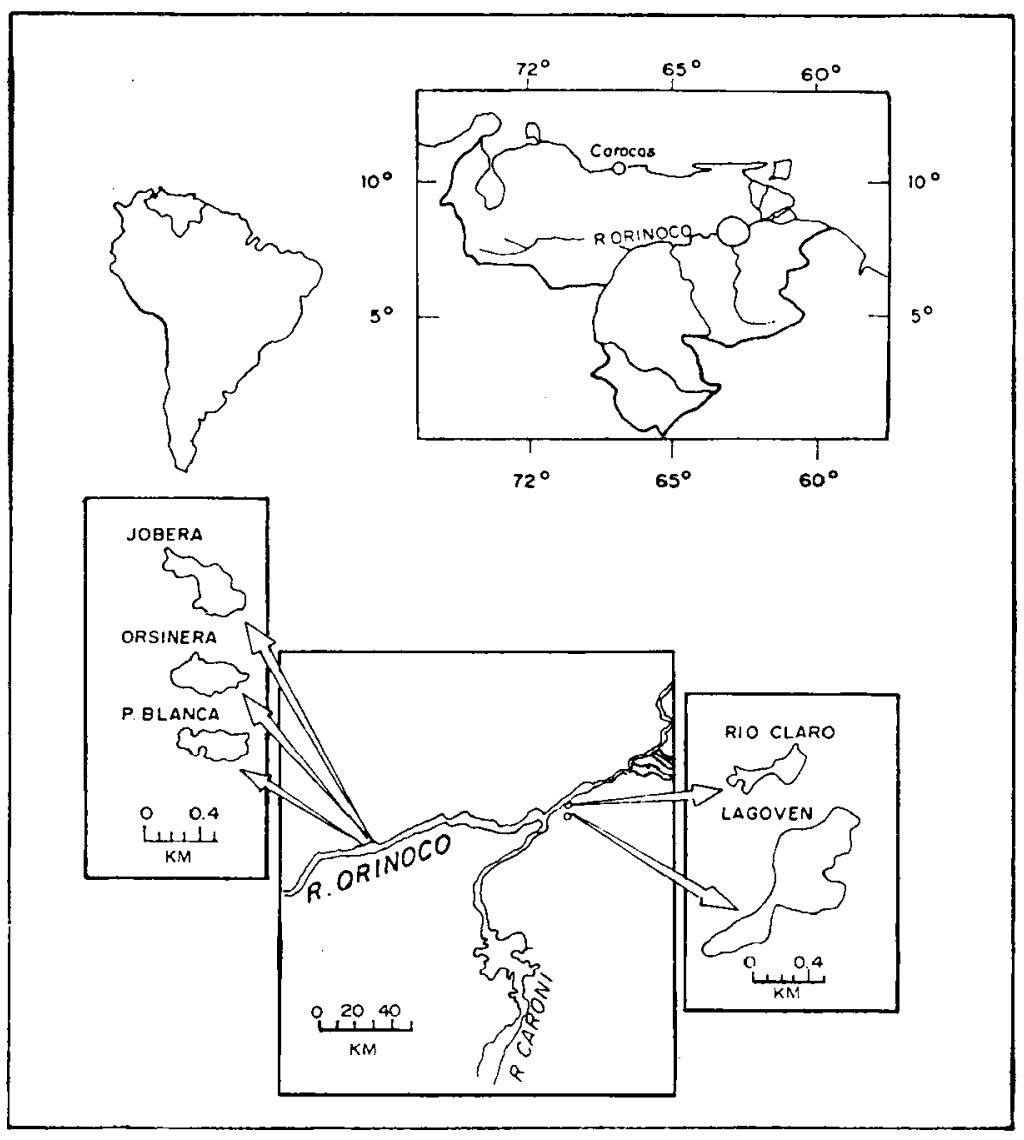

Fig. 1. Location of the study area. Approximate lake surfaces correspond to low water boundaries.

Fig. 1. Localisation de la région étudiée. L'évaluation des surfaces des lacs correspond aux périodes de basses eaux. 
Table 1. Morphometric data of the Orinoco lakes (Hw : high waters; Lw : low waters), (1: lenght ; b : breadth ; L : shore Line ; A : area ; DI : development of shore line ; Zmax. : maximum depth).

Tableau 1. Caractéristiques morphométriques de 5 lacs d'inondaion de l'Orénoque (H.w : hautes eaux; Lw : basses eaux), (I : longueur ; b : largeur ; L : périmètre ; A : surface ; Dl : développement de la ligne de côte ; Zmax. : profondeur maximum).

\begin{tabular}{|c|c|c|c|c|c|c|c|c|c|c|c|c|c|c|}
\hline & \multicolumn{2}{|c|}{$\mathrm{I}(\mathrm{m})$} & \multicolumn{2}{|c|}{$b(m)$} & \multicolumn{2}{|c|}{$L(m)$} & \multicolumn{2}{|c|}{$A(h a)$} & \multicolumn{2}{|c|}{ DI } & \multicolumn{2}{|c|}{ l:b } & \multicolumn{2}{|c|}{$Z \max .(m)$} \\
\hline & $\mathrm{Hw}$ & $\mathrm{Lw}$ & $\mathrm{Hw}$ & Lw & $\mathrm{Hw}$ & Lw & $\mathrm{Hw}$ & $\mathrm{Lw}$ & $\mathrm{Hw}$ & Lw & $\mathrm{Hw}$ & Lw & $\mathrm{Hw}$ & Lw \\
\hline P. Blanca & 690 & 450 & 210 & 200 & 1900 & 1500 & 9.6 & 5.1 & 1.73 & 1.87 & 3.29 & 2.25 & 4.2 & 0.3 \\
\hline Orsinera & 640 & 590 & 300 & 280 & 1500 & 1400 & 12.1 & 11.5 & 1.22 & 1.17 & 2.13 & 2.11 & 5.8 & 1.2 \\
\hline Jobera & 670 & 560 & 470 & 360 & 2300 & 1600 & 14.7 & 10.1 & 1.69 & 1.42 & 1.43 & 1.56 & 4.8 & 0.6 \\
\hline Lagoven & 1120 & 820 & 660 & 580 & 4400 & 2400 & 40.9 & 29.3 & 1.94 & 1.25 & 1.70 & 1.41 & 4.5 & 0.9 \\
\hline A. Claro & 520 & 260 & 200 & 140 & 1200 & 600 & 5.0 & 1.4 & 1.51 & 1.44 & 2.60 & 1.86 & 2.4 & 0.1 \\
\hline
\end{tabular}

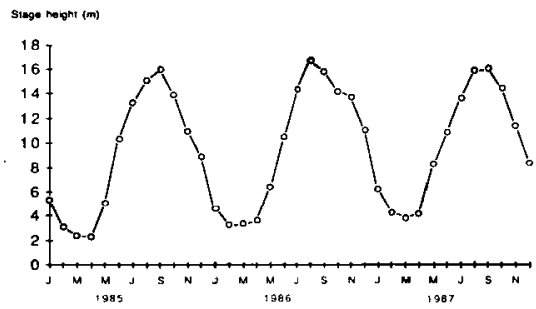

Fig. 2. Stage height in the Orinoco River (Angostura), (1985-1987).

Fig. 2. Variation du niveau de l'Orénoque (1985-1987).
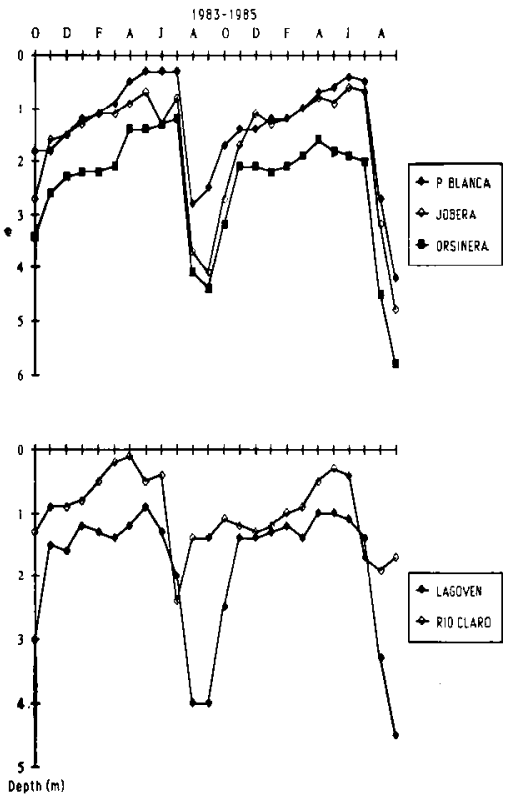

Fig. 3. Water depth variations in the Orinoco lakes.

Fig. 3. Variation de la hauteur d'eau dans 5 lacs d'inondation de l'Orénoque. 


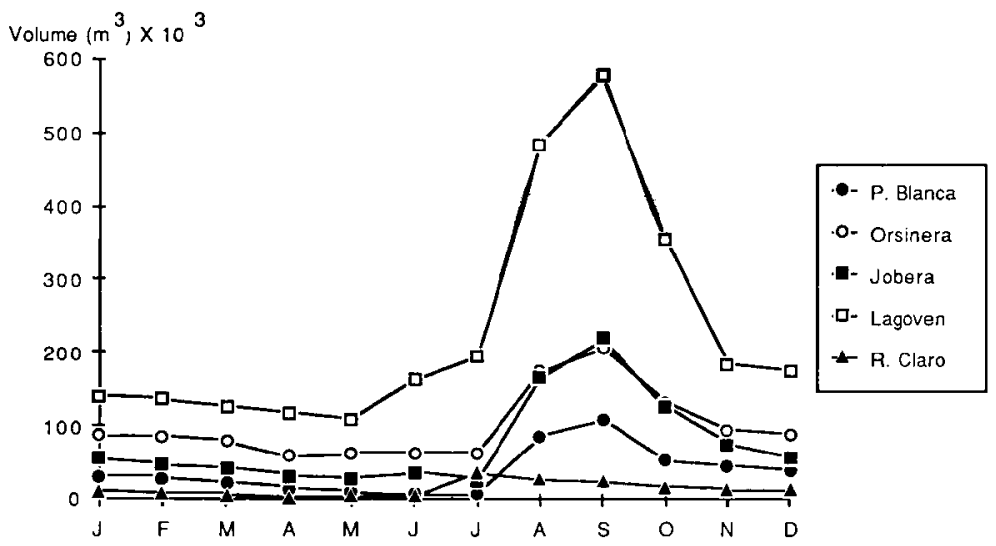

Fig. 4. Estimates of water volumes in the Orinoco lakes (mean monthly values of the 1983-1985 period).

Fip 4. Estimation des yolumes d'eau dans 5 lacs d'inondation de l'Orénoque. (Valeurs moyennes mensuelles pour la période 1983-1985).

of a cone. For monthly changes in lake surfaces, we used planimetric data from high and low waters and assumed, using these two extremes, similar decrements in lake surfaces. Depth values were used in the estimates obtaining the monthly mean of a 24-month period. Given the limitations of these estimates they are used only to give an idea of lake volumes.

Data on water depth, Secchi disc transparency, conductivity, dissolved oxygen, and water temperature are given in Table 2. Mean transparency in lakes was $32.8 \mathrm{~cm} \pm 7.5$; mean conductivity was $106.8 \pm 107.9 \mu \mathrm{S} / \mathrm{cm}$. L. Río Claro showed higher conductivity values (mean : $295.7 \mu \mathrm{S} / \mathrm{cm}$ ) compared to a mean of $59.6 \mu \mathrm{S} / \mathrm{cm}$ for the remaining lakes. Seasonally, lakes transparency was highest during inundation while conductivity generally showed high values at low waters. Low transparency and high conductivity at low waters are the result of bottom sediments resuspension when lakes are shallowest. Vásquez (1992) classified these lakes as continuous warm polymictic. Dissolved oxygen shows a highly significant negative relationship with water depth. The lakes are generally below dissolved oxygen saturation.

\section{Materials and methods}

Monthly zooplankton collection were made over a 21-month period (Junuary 1984 - September 1985) at sites over the deepest part of the lakes in areas free of macrophyte beds. A variable volume of subsurface water (20-40 l) was filtered through a net of $45 \mu \mathrm{m}$ mesh. For counting, $5 \mathrm{ml}$ subsamples were examined. This volume generally allowed the identification of at least 100 of the most common species or stages. When density was low, the entire sample was usually examided. Rose bengal was added to the samples to facilitate separation of organisms from suspended matter.

Counting of rotifers and cladocerans was done to the species level. Copepods were counted as developmental stages (adult cyclopoids, adult calanoids, copepodites, and nauplii). Rotifers and cladocerans 
Table 2. Ranges, mean and standard deviations of depth, transparency, conductivity (at $25^{\circ} \mathrm{C}$ ), dissolved oxygen and temperature in the Orinoco lakes.

Tableau 2. Valeur minimale, maximale, moyenne et déviation standard de la profondeur, de la transparence, de la conductivité (a $25^{\circ} \mathrm{C}$ ), de l'oxygène dissous et de la température dans 5 lacs d'inondation de l'Orénoque.

\begin{tabular}{|c|c|c|c|c|}
\hline & Min & $\operatorname{Max}$ & Mean & sd \\
\hline $\begin{array}{l}\text { P. Blanca } \\
\text { Depth (m) } \\
\text { Transparency }(\mathrm{cm}) \\
\text { Conductivity }(\mu \mathrm{S} / \mathrm{cm}) \\
\text { DO (mg/l) } \\
\text { Temperature }\left({ }^{\circ} \mathrm{C}\right)\end{array}$ & $\begin{array}{r}0.3 \\
5.0 \\
22.0 \\
1.8 \\
27.0\end{array}$ & $\begin{array}{r}4.2 \\
120.0 \\
83.0 \\
7 \% \\
825\end{array}$ & $\begin{array}{r}1.3 \\
42.2 \\
43.7 \\
5.3 \\
29.7\end{array}$ & $\begin{array}{r}0.96 \\
31.76 \\
14.56 \\
1.73 \\
1.85\end{array}$ \\
\hline $\begin{array}{l}\text { Orsinera } \\
\text { Depth }(\mathrm{m}) \\
\text { Transparency }(\mathrm{cm}) \\
\text { Conductivity }(\mu \mathrm{S} / \mathrm{cm}) \\
\text { DO (mg/l) } \\
\text { Temperature }\left({ }^{\circ} \mathrm{C}\right)\end{array}$ & $\begin{array}{r}1.2 \\
5.0 \\
21.0 \\
3.1 \\
26.0\end{array}$ & $\begin{array}{r}5.8 \\
120.0 \\
56.6 \\
7.6 \\
\mathbf{3 1 . 5}\end{array}$ & $\begin{array}{r}2.5 \\
36.4 \\
35.1 \\
5.8 \\
29.0\end{array}$ & $\begin{array}{r}1.16 \\
29.10 \\
8.80 \\
1.09 \\
1.67\end{array}$ \\
\hline $\begin{array}{l}\text { Jobera } \\
\text { Depth }(\mathrm{m}) \\
\text { Transparency }(\mathrm{cm}) \\
\text { Conductivity }(\mu \mathrm{S} / \mathrm{cm}) \\
\text { DO (mg/l) } \\
\text { Temperature }\left({ }^{\circ} \mathrm{C}\right)\end{array}$ & $\begin{array}{r}0.6 \\
5.0 \\
28.0 \\
3.9 \\
26.5\end{array}$ & $\begin{array}{r}4.8 \\
75.0 \\
196.0 \\
7.8 \\
31.0\end{array}$ & $\begin{array}{r}1.7 \\
27.4 \\
67.3 \\
5.8 \\
29.2\end{array}$ & $\begin{array}{r}1.18 \\
20.45 \\
38.81 \\
1.11 \\
1.23\end{array}$ \\
\hline $\begin{array}{l}\text { Lagoven } \\
\text { Depth }(\mathrm{m}) \\
\text { Transparency }(\mathrm{cm}) \\
\text { Conductivity }(\mu \mathrm{S} / \mathrm{cm}) \\
\text { DO (mg/l) } \\
\text { Temperature }\left({ }^{\circ} \mathrm{C}\right)\end{array}$ & $\begin{array}{r}0.9 \\
10.0 \\
24.0 \\
4.0 \\
26.0\end{array}$ & $\begin{array}{r}4.5 \\
93.0 \\
261.0 \\
7.8 \\
31.5\end{array}$ & $\begin{array}{r}1.9 \\
35.0 \\
92.3 \\
6.1 \\
29.1\end{array}$ & $\begin{array}{r}1.07 \\
25.36 \\
64.40 \\
0.89 \\
1.34\end{array}$ \\
\hline $\begin{array}{l}\text { R. Claro } \\
\text { Depth (m) } \\
\text { Transparency }(\mathrm{cm}) \\
\text { Conductivity }(\mu \mathrm{S} / \mathrm{cm}) \\
\text { DO }(\mathrm{mg} / \mathrm{l}) \\
\text { Temperature }\left({ }^{\circ} \mathrm{C}\right)\end{array}$ & $\begin{array}{r}0.1 \\
5.0 \\
93.0 \\
4.6 \\
21.5\end{array}$ & $\begin{array}{r}2.4 \\
53.0 \\
658.0 \\
9.8 \\
35.0\end{array}$ & $\begin{array}{r}1.0 \\
23.2 \\
295.7 \\
7.0 \\
29.5\end{array}$ & $\begin{array}{r}0.58 \\
15.44 \\
155.01 \\
1.41 \\
2.55\end{array}$ \\
\hline
\end{tabular}


demanding detailed taxonomic analysis were mounted on polyvinil alcohol and glicerine alcohol respectively.

\section{Results}

\subsection{Species composition and relative abundance}

Qualitative zooplankton analysis revealed the presence of 60 rotifer taxa. Brachionus and Keratella together accounted for $30,5 \%$ of the rotifers followed by species of Trichocerca $(18,6 \%)$ and Lecane $(13,6 \%)$. In spite of the high number of rotifera taxa, analysis of the specific frequency revealed that constant species $(\mathrm{C}>50 \%$ ) were few and mostly truly planktonic (Table 3 ). The number of constant species was 7 in all lakes except in L. Jobera which only showed 3 constant species. Dominant rotifer species included members of the Brachionidae plus a reduced group of species from the Synchaetidae and Filinidae.

In all lakes, rotifer species richness showed a mean value of $\mathbf{1 0 . 6}$ species (range : 9.3 species in $\mathrm{L}$. Playa Blanca - 11.6 species in L. Orsinera). The ratio of the number of species found at high water per lake was similar (Table 4). Richness per month and per lake, however, was generally higher during floods

The percentage of similitarity of the rotifer communities in all lakes was estimated by means of the index employed by Green (1972), Ps $=100-0.5 \Sigma$ $|\mathrm{a}-\mathrm{b}|$, where " $\mathrm{a}$ " is the relative abundance in the inventory $A$ and $\alpha \mathrm{b}$ ) is the relative abundance of the same species in the inventory B, both of them expressed in terms of the total percentage. Mean similarity among lakes was very similar for both high and low water periods ( $\mathrm{Ps}=43,7 \%$, low waters ;
Ps $=43,9 \%$, high waters) (Table 5). As suggested by Paggi \& José de Paggi (i980), a high similarity in species composition should be expected in proximate lakes from a same hydrographic basin, with similar geological characteristics and highly influenced by a common source of water during floods.

In terms of mean abundance, rotifers were always more abundant than copepods and cladocerans (Fig. 5). Mean abundance of rotifers in all lakes accounted for $73,4 \%$ of total zooplankton (L. Rio Claro : 95,5\% ; L. Jobera : 78,9\% ; L. Playa Blanca : $73,4 \%$; L. Orsinera : $63,2 \%$; and L. Lagoven : 56,2 \%). Common abundant species included mainly euplanktonic species such as Keretella americana, $K$. cochlearis, Brachionus mirus, B. gessneri, Polyarthra vulgaris and Filinia longiseta (Tables 6-10).

Table 3. Frequency of constant rotifer species $(\mathrm{C}>50 \%)$ in the Orinoco lakes.

Tableau 3. Fréquence des espèces constantes des rotifères $(C>50 \%)$ dans 5 lacs d'inondation de l'Orénoque.

\begin{tabular}{|c|c|c|c|c|c|}
\hline SPECIES & PB & OR & Jo & LG & RC \\
\hline$x$ americana & 83.5 & 85.0 & $8+.5$ & 66.5 & 87.5 \\
\hline B. gessneri & 66.0 & 68.0 & & 92.5 & 45.0 \\
\hline F. long I sets & 53.5 & 78.0 & & 75.0 & 76.0 \\
\hline e. mirus & & 83.5 & 76.0 & 81.5 & \\
\hline K. cochleeris & 61.5 & 71.0 & & 02.5 & \\
\hline P. vilgaris & & 050 & B6.5 & 86.5 & 52.0 \\
\hline B. anguleris & & 29.0 & & & \\
\hline$T$ sitmills & 51.0 & & & 630 & 66.0 \\
\hline A. fisso & & & & 61.5 & \\
\hline \multicolumn{6}{|l|}{ L protet } \\
\hline P. remete & 100.0 & & & 76.0 & \\
\hline \multicolumn{6}{|l|}{ P. lybera } \\
\hline T. stylala & 39.5 & & & & \\
\hline
\end{tabular}

Table 4. Mean species richness of rotifers at low waters (Lw), high waters (Hw), ratio Lw :Hw, and means for the period study.

Tableau 4. Richesse spécifique moyenne des rotifères à basses eaux ( $L w$ ) et hautes eaux (Hw) et valeurs moyennes pour la période d'étude.

\begin{tabular}{lrrrrr}
\hline Lakes & Lw & Hw & Lw:Hw & Mean & sd \\
\hline P. Blanca & 10.0 & 8.1 & 1.2 & 9.3 & 4.4 \\
Orsinera & 12.1 & 11.0 & 1.1 & 11.6 & 5.2 \\
Jobera & 8.7 & 12.4 & 0.7 & 10.1 & 4.6 \\
Lagoven & 10.6 & 10.9 & 1.0 & 10.7 & 3.8 \\
R. Claro & 11.7 & 10.0 & 1.2 & 11.1 & 5.8 \\
\hline
\end{tabular}



Abundance (\%)
Biomass (\%)
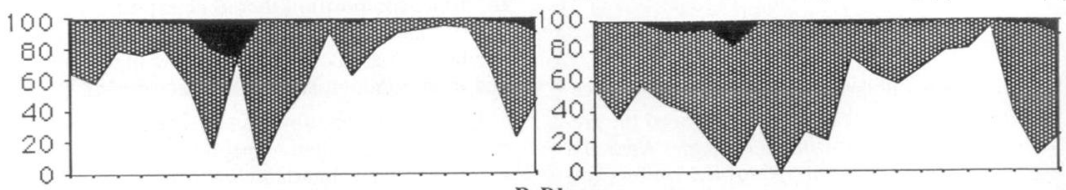

P. Blanca
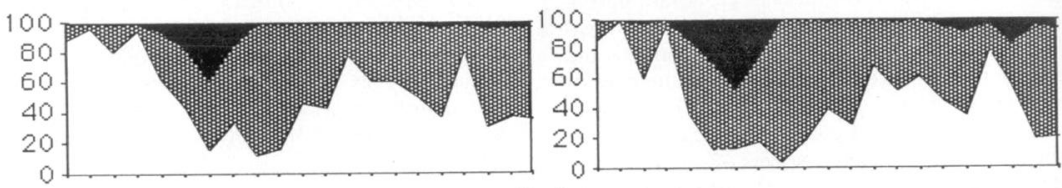

Orsinera
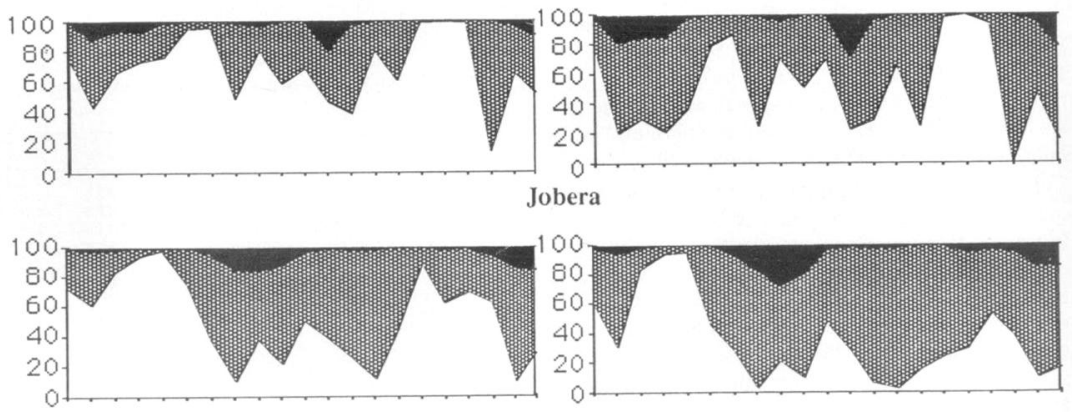

Lagoven
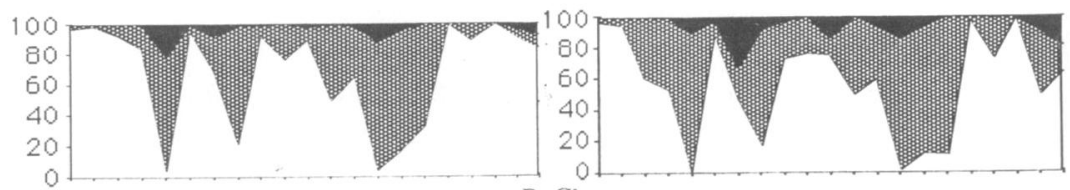

R. Claro

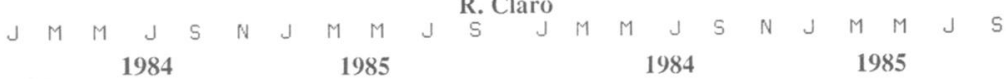

Fig. 5. Variations in the relative abundance and biomass of the major zooplankton groups in the Orinoco lakes. White : rotifers ; grey : copepods ; black : cladocerans.

Fig 5. Valeurs relatives de l'abondance et de la biomasse des principaux groupes de zooplancton dans 5 lacs d'inondation de l'Orénoque. Blanc : rotifères, gris : copépodes, noir : cladocères. 
Table 5. Percentage similarity of rotifers at low waters (a) and at high waters (b) in the Orinoco lakes.

Tableau 5. Pourcentage de similitude des rotifères à basses eaux (a) et à hautes eaux (b) dans 5 lacs d'inondation de l'Orenoque.

\begin{tabular}{|c|c|c|c|c|}
\hline & OR & J6 & $\mathrm{RC}$ & LG \\
\hline $\mathrm{PB}$ & 35.6 & 34.5 & 33.9 & 55.1 \\
\hline DR & & 39.3 & 35.6 & 55.6 \\
\hline J8 & & & 57.9 & 49.0 \\
\hline \multirow[t]{2}{*}{$\mathrm{RC}$} & & & & 40.0 \\
\hline & OR & J8 & $\mathrm{RC}$ & LG \\
\hline$P B$ & 47.8 & 45.5 & 26.1 & 58.5 \\
\hline OR & & 47.0 & 33.1 & 57.7 \\
\hline JB & & & 57.9 & 22.1 \\
\hline $\mathrm{RC}$ & & & & 43.0 \\
\hline
\end{tabular}

Table 6. Abundance of the common zooplankton species or developmental stages and biomass values of zooplankton groups in L. Playa Blanca.

Tableau 6. Abondance des espèces communes ou des stades de développernent des espèces du zooplancton, et valcurs de biomasse des groupes zooplanctonique dans le lac Playa Blanca.

\begin{tabular}{|c|c|c|c|c|c|c|}
\hline P. BLANCA & $\operatorname{Lw}$ & $H w$ & Lw:Hw & Mean & $\operatorname{Min}$ & $\operatorname{Max}$ \\
\hline \multicolumn{7}{|l|}{ DENSITY(org./) } \\
\hline ZOOPLANKTON: total & 640.0 & 144.3 & 4.4 & 451.1 & 4.0 & 2259.0 \\
\hline ROTIFERA: total & 487.3 & 77.1 & 6.3 & 331.1 & 2.0 & 2084.0 \\
\hline B. gessneri & 11.5 & 21.6 & 0.5 & 15.4 & 0.0 & 133.0 \\
\hline K. americana & 137.3 & 8.9 & 15.4 & 88.8 & 0.0 & 917.0 \\
\hline K. cochlearis & 176.8 & 12.0 & 14.7 & 114.0 & 0.0 & 1619.0 \\
\hline P. vulgaris & 103.9 & 2.5 & 41.6 & 65.2 & 0.0 & 478.0 \\
\hline \multicolumn{7}{|l|}{ COPEPODA } \\
\hline Adults ciclopoids & 16.0 & 5.4 & 3.0 & 11.9 & 0.0 & 100.0 \\
\hline Adults calanoids & 0.0 & 0.4 & - & 0.1 & 0.0 & 2.0 \\
\hline Copepodites & 25.6 & 8.8 & 2.9 & 19.2 & 0.0 & 172.0 \\
\hline Nauplii & 77.3 & 42.6 & 1.8 & 73.6 & 0.0 & 225.0 \\
\hline \multicolumn{7}{|l|}{ CLADOCERA } \\
\hline B. tubicen & 0.4 & 0.3 & 1.3 & 0.3 & 0.0 & 2.0 \\
\hline B. deitersi & 1.2 & 0.9 & 1.3 & 1.1 & 0.0 & 8.0 \\
\hline D. spinulosum & 4.2 & 0.1 & 42.0 & 2.6 & 0.0 & 33.0 \\
\hline M. minuta & 12.6 & 0.9 & 14.0 & 8.2 & 0.0 & 143.0 \\
\hline \multicolumn{7}{|l|}{ BIOMASS $(\mu g / 1)$} \\
\hline Rotifera & 56.1 & 16.3 & 3.4 & 40.9 & 0.1 & 212.9 \\
\hline Copepoda & 79.8 & 34.2 & 2.3 & 62.4 & 0.0 & 445.8 \\
\hline Cladocera & 10.8 & 1.2 & 9.0 & 7.1 & 0.0 & 92.5 \\
\hline
\end{tabular}


Table 7. Abundance of the common zooplankton species or developmental stages and biomass values of zooplankton groups in L. Orsinera.

Tableau 7. Abondance des especes communes ou des stades de développement des espèces du zooplancton, et valeurs de biomasse des groupes zooplanctoniques dans le lac Orsinera.

\begin{tabular}{|c|c|c|c|c|c|c|}
\hline ORSINERA & Lw & Hw & Lw:Hw & Mean & Min & $\operatorname{Max}$ \\
\hline \multicolumn{7}{|l|}{ DENSITY(org.I) } \\
\hline ZOOPLANKTON: total & 695.0 & 126.0 & 5.5 & 340.0 & 16.0 & 1569.0 \\
\hline ROTIFERA: total & 377.0 & 35.0 & 10.8 & 215.1 & 2.0 & 1147.0 \\
\hline B. gessneri & 103.0 & 0.0 & - & 38.8 & 0.0 & 283.0 \\
\hline B. mirus & 92.0 & 2.0 & 46.0 & 41.0 & 0.0 & 239.0 \\
\hline Conochilus & 0.0 & 4.0 & - & 0.9 & 0.0 & 10.0 \\
\hline F. longisela & 13.0 & 2.0 & 6.5 & 3.2 & 0.0 & 48.0 \\
\hline$H$. intermedia & 1.0 & 5.0 & 0.2 & 1.6 & 0.0 & 21.0 \\
\hline K. americana & 8.0 & 2.0 & 4.0 & 9.5 & 0.0 & 134.0 \\
\hline K. cochlearis & 0.0 & 3.0 & - & 0.8 & 0.0 & 9.0 \\
\hline L. proiecta & 30.0 & 1.0 & 30.0 & 34.1 & 0.0 & 498.0 \\
\hline P. vulgaris & 13.0 & 8.0 & 1.6 & 14.3 & 0.0 & 80.0 \\
\hline T. similis & 1.0 & 1.0 & 1.0 & 0.3 & 0.0 & 5.0 \\
\hline \multicolumn{7}{|l|}{ COPEPODA } \\
\hline Adults ciclopoids & 12.0 & 1.0 & 12.0 & 3.1 & 0.0 & 360 \\
\hline Adults calanoids & 0.1 & 1.0 & 0.1 & 0.2 & 0.0 & 2.0 \\
\hline Copepodites & 39.0 & 8.0 & 4.9 & 13.5 & 0.0 & 106.0 \\
\hline Nauplī & 265.0 & 74.0 & 3.6 & 102.4 & 12.0 & 693.0 \\
\hline \multicolumn{7}{|l|}{ CLADOCERA } \\
\hline B. tubicen & 0.2 & 1.0 & 0.2 & 0.5 & 0.0 & 5.0 \\
\hline B. deitersi & 0.1 & 0.7 & 0.1 & 0.2 & 0.0 & 2.0 \\
\hline C. cornuta & 0.2 & 1.0 & 0.2 & 0.3 & 0.0 & 3.0 \\
\hline D. spinulosum & 1.0 & 0.3 & 4.0 & 0.1 & 0.0 & 2.0 \\
\hline M. minuta & 3.0 & 8.0 & 0.4 & 4.5 & 0.0 & 35.0 \\
\hline \multicolumn{7}{|l|}{ BIOMASS $(\mu g /)$} \\
\hline Rotifera & 95.0 & 8.0 & 11.9 & 52.9 & 0.2 & 295.6 \\
\hline Copepoda & 110.0 & 18.0 & 6.1 & 35.4 & 2.0 & 298.0 \\
\hline Cladocera & 2.0 & 6.0 & 0.3 & 3.3 & 0.0 & 23.7 \\
\hline
\end{tabular}

Copepod species included the cyclopoids Mesocyclops sp., Oithona amazonica, Thermocyclops minutus, and $T$. decipiens and the calanoids Diaptomus negrensis, Notodiaptomus amazonicus, $N$. caerensis, and Rhacodiaptomus calatus. All developmental copepod stages were observed, nauplii, howewer, comprised the highest proportion among copepods. Mean abundance of nauplii with respect to the other copepod stages was: $\mathbf{L}$. Orsinera : $85.9 \%$; L. Jobera : $75.6 \%$; L. Lagoven : $72.6 \%$; L.P. Blanca : $70.2 \%$ and L.R. Claro : $64.5 \%$ (Fig. 5). Highest mean abundance of adult copepods was due to cyclopoids except in L. R. Claro where calanoids were more abundant (Tables 6-10).

Cladocerans from these lakes and other lentic and lotic water bodies were investigated by Rey \& Wásquez (1986). These authors identified 7 pelagic and 25 littoral species (mainly Chydoridae) from floodplain lakes of the Orinoco. In the present study, cladocerans were dominated by planktonic species (mainly Moina minuta, Ceriodaphnia cornuta and Diaphanosoma spinulosum (Tables 6-10). $M$. minuta accounted for almost $60 \%$ of the mean 
Table 8. Abundance of the common zooplankton species or developmental stages and biomass values of zooplankton groups in $[$. Jobera.

Tableau 8. Abondance des espèces ommunes ou des stades de développement des espèces du zooplanct on, et valeurs de biomasse des groupes zooplanctoniques dans le lac Jobera.

\begin{tabular}{|c|c|c|c|c|c|c|}
\hline JOBERA & Lw & $H w$ & $L w: H w$ & Mean & Min & $\operatorname{Max}$ \\
\hline \multicolumn{7}{|l|}{ DENSITY(org.fl) } \\
\hline ZOOPLANKTON: total & 417.7 & 276.8 & 1.5 & 364.0 & 38.0 & 1286.0 \\
\hline ROTIFERA: total & 343.1 & 195.9 & 1.8 & 287.0 & 5.0 & 1222.0 \\
\hline A. navicula & 0.0 & 65.3 & - & 24.9 & 0.0 & 499.0 \\
\hline A. saltans & 0.0 & 4.0 & - & 1.5 & 0.0 & 32.0 \\
\hline B. gessneri & 3.7 & 17.8 & 0.2 & 9.0 & 0.0 & 55.0 \\
\hline B. mins & 10.9 & 0.0 & - & 6.8 & 0.0 & 52.0 \\
\hline Conochilus sp. & 0.0 & 16.1 & - & 6.1 & 0.0 & 129.0 \\
\hline K. americana & 269.4 & 5.6 & 48.1 & 168.9 & 0.0 & 1054.0 \\
\hline K. cochlearis & 1.3 & 4.3 & 0.3 & 2.4 & 0.0 & 33.0 \\
\hline K. nhamunda & 0.0 & 21.4 & - & 8.1 & 0.0 & 121.0 \\
\hline P. vilgaris & 2.1 & 1.8 & 1.2 & 2.0 & 0.0 & 19.0 \\
\hline \multicolumn{7}{|l|}{ COPEPODA } \\
\hline Adults ciclopoids & 3.8 & 4.8 & 0.8 & 4.2 & 0.0 & 16.0 \\
\hline Adults calanoids & 0.9 & 2.7 & 0.3 & 1.6 & 0.0 & 17.0 \\
\hline Copepodites & 10.9 & 10.8 & 1.0 & 10.9 & 0.0 & 47.0 \\
\hline Nauplii & 47.0 & 59.3 & 0.8 & 51.7 & 1.0 & 198.0 \\
\hline \multicolumn{7}{|l|}{ CLADOCERA } \\
\hline B. tubicen & 0.4 & 0.0 & & 0.2 & 0.0 & 2.0 \\
\hline B. deitersi & 0.1 & 2.0 & 0.05 & 0.8 & 0.0 & 6.0 \\
\hline C. cornuta & 5.4 & 0.1 & 54.0 & 3.4 & 0.0 & 70.0 \\
\hline M. minuta & 5.9 & 1.5 & 3.9 & 4.2 & 0.0 & 37.0 \\
\hline \multicolumn{7}{|l|}{ BIOMASS $(\mu g / 1)$} \\
\hline Rotifera & 36.4 & 29.3 & 1.2 & 33.7 & 0.5 & 111.3 \\
\hline Copepoda & 29.5 & 37.0 & 0.8 & 32.4 & 0.3 & 113.7 \\
\hline Cladocera & 7.0 & 1.7 & 4.1 & 5.0 & 0.0 & 51.2 \\
\hline
\end{tabular}

cladoceran abundance in all lakes. In general, species richness and mean abundance of cladocerans was relatively low as previously reported by Rey and Vásquez (1986).

\subsection{Patterns of abundance and biomass}

Mean zooplankton density in the lakes showed the following sequence : R: Claro $>$ P. Blanca $>$ Jobera $>$ Lagoven $>$ Orsinera. Excluding L. Rio Claro, mean zooplankton density was 375.4 ind./l. Lake Rio Claro showed almost a 10 -fold increase in its mean zooplankton density compared to the other lakes.
Analysis of the mean zooplankton density at low and high waters revealed highest mean density values during the low water period for most lakes. The ratio of low water mean density to high water mean density ranged between 1.0 and 43.7 and showed the following sequence : Río Claro $>$ Orsinera $>$ P. Blanca > Jobera. L. Lagoven showed a ratio close to 1 indicating similar mean density values for both low and high water periods.

Estimates of zooplankton population sizes from densities and lake volumes showed highest values during inundation in lakes Lagoven, Orsinera, and Jobera. Lakes P. Blanca and R. Claro showed, 
Table 9. Abundance of the common zooplankton species or developmental stages and biomass values of zooplankton groups in L. Lagoven.. Tableau 9. Abondance des espèces communes ou des stades de développement des espèces du zooplancton, et valeurs de biomasse des groupes zooplanctoniques dans le lac Lagoven.

\begin{tabular}{|c|c|c|c|c|c|c|}
\hline LAGOVEN & $\mathbf{L w}$ & $\mathrm{Hw}$ & Lw:Hw & Mean & Min & Max \\
\hline \multicolumn{7}{|l|}{ DENSITY(org./I) } \\
\hline ZOOPLANKTON: total & 340.5 & 356.2 & 1.0 & 346.5 & 9.0 & 985.0 \\
\hline ROTIFEAA: total & 215.2 & 161.7 & 1.3 & 194.8 & 8.0 & 603.0 \\
\hline B. mins & 4.9 & 5.3 & 0.9 & 5.1 & 0.0 & 57.0 \\
\hline B. zahniseri & 0.0 & 2.6 & - & 1.0 & 0.0 & 11.0 \\
\hline F. longiseta & 0.0 & 3.5 & - & 1.3 & 0.0 & 19.00 \\
\hline K. americana & 75.5 & 42.5 & 1.8 & 62.9 & 0.0 & 355.0 \\
\hline K. cochlearis & 33.7 & 32.3 & 1.0 & 33.1 & 0.0 & 247 \\
\hline P. vulgaris & 13.6 & 9.0 & 1.5 & 11.9 & 0.0 & 114.0 \\
\hline \multicolumn{7}{|l|}{ COPEPODA } \\
\hline Adults ciclopoids & 7.9 & 7.3 & 1.1 & 7.6 & 0.0 & 25.0 \\
\hline Adults calanoids & 0.4 & 3.3 & 0.1 & 1.5 & 0.0 & 19 \\
\hline Copepodites & 29.0 & 28.8 & 1.0 & 28.9 & 0.0 & 80.0 \\
\hline Nauplii & 82.7 & 130.4 & 0.6 & 100.9 & 0.0 & 253.0 \\
\hline \multicolumn{7}{|l|}{ CLADOCERA } \\
\hline B. tubicen & 1.5 & 0.4 & 3.8 & 1.0 & 0.0 & 11.0 \\
\hline B. deitersi & 0.5 & 7.0 & 0.07 & 3.0 & 0.0 & 18.0 \\
\hline C. cornuta & 0.0 & 5.4 & - & 2.1 & 0.0 & 26.0 \\
\hline D. spinulosum & 0.1 & 4.1 & 0.02 & 1.6 & 0.0 & 29.0 \\
\hline M. minuta & 3.4 & 8.1 & 0.4 & 5.2 & 0.0 & 17.0 \\
\hline \multicolumn{7}{|l|}{ BIOMASS ( $\left.\mu g^{\prime}\right)$} \\
\hline Rotifera & 40.3 & 29.8 & 1.4 & 36.3 & 0.9 & 92.1 \\
\hline Copepoda & 58.9 & 68.3 & 0.9 & 62.3 & 2.8 & 170.2 \\
\hline Cladocera & 3.1 & 12.5 & 0.3 & 6.7 & 0.0 & 33.6 \\
\hline
\end{tabular}

however, highest population densities at low waters (Table 2). Comparison of morphometric data from both high and low waters showed that lakes $P$. Blanca and R. Claro presented the highest variability in lake surfaces and depths. The ratio of high water to low water surface was almost 2 for L. P. Blanca and 3.6 for L. R. Claro. The ratio of water depth was 14 for L. P. Blanca and 24 for L. R. Claro. These drastic changes in surface and water depth coupled with high zooplankton densities may explain the highest mean population sizes recorded at low water in the these two lakes as a consequence of high concentration of zooplankton organisms.

In terms of biomass, mean dry mass in all lakes was $162.2 \mu \mathrm{g} / \mathrm{l}$ ranging from $71.1 \mu \mathrm{g} /$ to $432.8 \mu \mathrm{g} / \mathrm{l}$.
The following sequence was observed : R. Claro > P. Blanca $>$ Lagoven $>$ Orsinera $>$ Jobera. In lakes Orsinera, Jobera, and R. Claro, rotifers accounted for $64.7 \%$ of the total mean zooplankton biomass. In lakes P. Blanca and Lagoven, however, copepods showed highest mean biomass values $(57.8 \%)$.

Seasonally, the ratio of mean low water zooplankton biomass to mean high water biomass revealed highest mean biomass values at low waters. The mean overall ratio was 6.5 ranging from 0.9 to 18.4 . These figures indicate that seasonality of biomass is a conspicuous characteristic of the lakes (Tables 6-10, Fig. 5). The ratio showed the following sequence : R. Claro $>$ Orsinera $>$ P. Blanca $>$ Jobera > Lagoven. 
Table 10. Abundance of the common zooplankton species or developmental stages and biomass values of zooplankton groups in L. R. Claro.

Tableau 10. Abondance des espèces ommunes ou des stades de développernent des espéces du zooplancton, et valeurs de biomasse des groupes zooplanctoniques dans le lac Rio Claro.

\begin{tabular}{|c|c|c|c|c|c|c|}
\hline RIO CLARO & $L w$ & $\mathrm{Hw}$ & Lw:Hw & Mean & Min & Max \\
\hline \multicolumn{7}{|l|}{ DENSITY(org $/ I)$} \\
\hline ZOOPLANKTON: total & 5553.0 & 1270.0 & 43.7 & 3486.1 & 7.5 & 49430.0 \\
\hline ROTIFERA: total & 5316.5 & 95.1 & 55.9 & 3327.4 & 0.4 & 49078.0 \\
\hline B. angularis & 24.1 & 11.0 & 2.2 & 19.1 & 0.0 & 313.0 \\
\hline B. caudatus & 19.0 & 0.0 & - & 11.8 & 0.0 & 125.0 \\
\hline B. havanaensis & 13.2 & 0.2 & 82.7 & 8.3 & 0.0 & 96.0 \\
\hline F. longiseta & 9.9 & 1.5 & 6.6 & 6.7 & 0.0 & 123.0 \\
\hline K. americana & 4438.2 & 1.6 & 2722.8 & 2748.1 & 0.0 & 45378.0 \\
\hline K. tropica & 183.7 & 9.6 & 19.1 & 117.4 & 0.0 & 2388.0 \\
\hline L. proiecta & 111.7 & 0.1 & 859.2 & 69.2 & 0.0 & 1445.0 \\
\hline P. vilgaris & 19.8 & 4.3 & 4.7 & 13.9 & 0.0 & 135.0 \\
\hline P. libera & 0.1 & 0.4 & 0.1 & 0.2 & 0.0 & 3.0 \\
\hline \multicolumn{7}{|l|}{ COPEPODA } \\
\hline Adults ciclopoids & 7.9 & 0.1 & 60.8 & 4.9 & 0.0 & 48.0 \\
\hline Adults calanoiós & 14.3 & 0.0 & - & 8.9 & 0.0 & 163.0 \\
\hline Copepodites & 62.2 & 0.4 & 155.5 & 38.6 & 0.0 & 316.0 \\
\hline Nauplii & 135.3 & 30.4 & 4.5 & 95.3 & 0.0 & 692.0 \\
\hline \multicolumn{7}{|l|}{ CLADOCERA } \\
\hline B. tubicen & 0.02 & 0.0 & - & C.01 & 0.0 & 0.2 \\
\hline B. deitersi & 0.2 & 0.04 & 3.8 & 0.1 & 0.0 & 1.0 \\
\hline D. spinulosum & 8.2 & 0.1 & 63.4 & 5.2 & 0.0 & 100.0 \\
\hline M. minuta & 8.4 & 0.1 & 64.8 & 5.3 & 0.0 & 105.0 \\
\hline 1. spinifer & 0.5 & 0.3 & 1.8 & 0.4 & 0.0 & 3.0 \\
\hline \multicolumn{7}{|l|}{ BIOMASS $(\mu g /)$} \\
\hline Rotifera & 595.5 & 15.8 & 37.6 & 384.5 & 0.1 & 5331.4 \\
\hline Copepoda & 72.5 & 5.7 & 12.7 & 47.0 & 0.0 & 149.6 \\
\hline Cladocera & 1.9 & 0.4 & 4.9 & 1.3 & 0.0 & 11.1 \\
\hline
\end{tabular}

Table 11. Estimates of zooplankton population sizes (ind. $\times 10^{6}$ ) in the Orinoco lakes.

Tableau 11. Estimation de la taille des populations zooplanctoniques (ind. $\times 10^{6}$ ) dans 5 lacs d'inondation de l'Orénoque.

\begin{tabular}{lrrrrrr}
\hline Lakes & Hw & Lw & Hw:Lw & Mean & Min & Max \\
\hline P. Blanca & 6868 & 12707 & 0.54 & 10483 & 337 & 87021 \\
Orsinera & 32642 & 27199 & 1.20 & 29532 & 3278 & 146257 \\
Jobera & 31186 & 15927 & 1.96 & 21740 & 960 & 86007 \\
Lagoven & 111736 & 45799 & 2.44 & 70918 & 1047 & 188850 \\
R. Claro & 2339 & 19675 & 0.12 & 13071 & 67 & 87876 \\
\hline
\end{tabular}




\section{Discussion}

The zooplankton of Orinoco floodplain lakes frequently included a mean of 7 constant rotifer species, and one or two species of Moina, Ceriodaphnia and Diaphanosoma. The number of species by water body for a given moment was from 1 to 24 rotifers, and 0 to 5 cladocerans. Including the overwhelming abundance of nauplii stages over other copepod stages, most zooplankton from the lakes were euplanktonic with a predominance of filter feeders and microphagous detritivores. This general picture of zooplankton composition and main feeding regimes of zooplankton of Orinoco lakes resembles that observed in lakes of other tropical and subtropical floodplain systems, such as the Niger in Africa (Dumont 1986) ; the Amazon and Parana in South America (Robertson \& Hardy 1984, Paggi \& José de Paggi 1990) and the Murray-Darling in Australia (Shiel 1986).

Compared to the Orinoco River main stem (Vásquez \& Rey 1989), both the river and the lakes show a numerically rich rotiferan fauna with a group of few, abundant and frequent species, relative scarcity of cladocerans and dominance of nauplii among copepod stages. In the lakes the association of dominant rotifer species is largely composed of euplanktonic forms while, as expected, the river shows a dominance of euplanktonic and tychoplanktonic forms. The most typical cladoceran association in the lakes was formed by $M$. Minuta, C. cornuta and $D$. spinulosum. In the river dominant species included $B$. deitersi, $B$. tubicen, $M$. minuta and $M$. reticulata.

The dominance of a core group of euplanktonic zooplankton species plus some other morphological and chemical features of orinoco lakes (high water level fluctuations in relation to lake volume, diurnal and annual variations in dissolved oxygen content) represent common features to the shallow lakes category of stagnant waters proposed by Higler \& Statzner (1988). Orinoco lakes, however, may reach water depth values similar to the category of very shallow lakes.

Compared to mean density of zooplankton in the Orinoco main stem (Saunders \& Lewis 1989, Vásquez \& Rey 1989), mean density in the lakes was 100 times higher than in the river. A similar value was reported by Hamilton et al. (1990) from another Orinoco floodplain lake. Considering the extension of the floodplain and the high abundance of zooplankton found in these areas, the contribution of zooplankton biomass from floodplain areas to the river is very small (Saunders \& Lewis 1989). Hamilton et al. (1990) ascribed the absence of a significant export of zooplankton as a consequence of retention of planktonic organisms by aquatic macrophytes. Reduced hydraulic washout due to reduced flow in floodplain areas as a consequence of flow atenuation in forested areas may also be important factors reducing the likelihood of zooplankton export. For exemple, Neiff et al. (in press) found a reduction of flow in the lower Paraguay during inundation form $1.7 \mathrm{~m} / \mathrm{s}$ in the main stem to $0.09 \mathrm{~m} / \mathrm{s}$ in forested areas. Water movement inside the floodplain would be reduced by the " biological rugosity $»$ mainly imposed by the density and height of herbaceous vegetation which would disipate energy (Neiff et al. in press). The sheet flooding of the lakes would also lead to serial connections among the lakes in the path of flow (Lewis et al. 1990). These connections plus the retention of zooplankton organisms by macrophyte mats would most probably explain the reduced advective transport of zooplankton from floodplain areas to the river. Predation on zooplankton may also be an important factor involved in removal of organisms in transit. Preliminary data on stomach contents of several fish species from Orinoco lakes (V. Ponte, pers. com.) revealed a dominance of cladocerans and copepods in the stomachs of 21 fish species. In stagnant waters of the Apure River floodplain a similar observation has been made (E. Vásquez, unpublished). Twombly \& Lewis (1989) also suggested predation by fish and Chaoborus as the major factor controlling cladoceran abundance in an Orinoco lake.

We may summarize that reducted export of zooplankton biomass from floodplain waterbodies to the river may be directly or indirectly controlled by physical (serial connections among lakes, high hydraulic residence times) and biological factors (retention of zooplankton by vegetation, predation).

Both in terms of abundance per unit volume of water and in terms of population sizes, zooplankton of Orinoco lakes was markedly seasonal. Mean annual density and biomass values allowed us to rank the lakes in the following order : density : RC $>\mathrm{PB}>\mathrm{JOB}>\mathrm{LAG}>\mathrm{ORS}>$; biomass : 
$\mathrm{RC}>\mathrm{PB}>\mathrm{LAG}>\mathrm{ORS}>\mathrm{JOB}$. Excluding L. Rio Claro, mean zooplankton abundance and biomass values in the remaining four lakes were very similar (mean density : $375.4 \pm 51.5$ ind. $/ 1$; mean biomass : $94.6 \pm 17.6 \mu \mathrm{g} / \mathrm{l})$. The peculiarities of $\mathrm{L}$. R. Claro which may help interpret its higher zooplankton density ( $\sim 10$ times higher) may be due to the fact this lake is seasonally inundated by waters from the Orinoco and Upata rivers. The latter intermitent river drains agricultural areas and we could expect a high organic load in its waters. The peculiarities of L. Rio Claro basin also include very hight conductivity values with respect to the other lakes ( $\sim 5$ times higher), and a much higher phytoplankton density (L. Sánchez, pers. com).

Analysis of the ratios of high water over low water lake surfaces and lake water depths revealed the same sequence for both parameters $(\mathrm{RC}>\mathrm{PB}>$ JOB > LAG > ORS) which corresponds to the sequence of zooplankton density. In other words, in lakes with highest variabilities in surface and water depths, zooplankton abundance was higher. Increased variations in surfaces and water depths imply higher water-land interactions in the eulittoral zones of the lakes. Breen et al. (1978) consider water depth, water residence time and the extension of inundated areas, of particular importance to the biological productivity of floodplain lakes. These factors would determine inputs of organic and inorganic material from the margins into the lakes leading to increased levels of food available for zooplankton.

From the previous information, we may summarize that in terms of zooplankton species composition, Orinoco lakes show a high degree of similarity among themselves. The observed differences in terms of density and biomass seem to indicate, however, that morphometry of each lake basin is important to explain differences in zooplankton productivity. Lakes with highest variability both in lake surfaces and water depths seem to support highest zooplankton abundance and biomass.

When an indirect connection is established with the major source of waters, the particular characteristics of the connector also seem important to interpret productivity. This may be the case of $\mathrm{L}$. Río Claro which is seasonally connected to the Orinoco river by the regressive inundations of the Upata River rich in electrolytes and organic load.

\section{Acknowledgements}

We are grateful to Dr. C. H. Fernando (Waterloo, Ontario, Canada) for his collaboration in the identification of copepods. We are also deeply grateful to Ms. M-L. Medina and Mr. J. C. Nasser for their support in the analytical work.

\section{References}

Breen C.M., Fumess H.D., Heeg J. \& Kok J. 1978. Bathymetric studies on the Pongolo River floodplain. J. Limnol. Soc. South. Africa., $4: 95-100$.

Drago E. 1976. - Origen y clasificación de ambientes leníticos en llanuras aluviales. Rev. Asoc. Cienc. Nal. Lit., $7:$ 123-137.

Drago E. 1981. - Grados de conexión y fases hidrologicas en ambientes leniticos de la llanura aluvial del ró Paraná (Argentina). Ecología (Argentina), $6: 27-33$.

Dumont H.J. 1986. - Zooplankton of the Niger system. In Davies, B. R. \& Walker, K.F. (eds.), The Ecology of River Systems. Monogr. Biol. no. 60, Junk, Dordrecht, 49-59.

Green J. 1972. - Freshwater ecology in the Mato Grosso. III. Associations of Rotifera in meander lakes of the Rio Suiá Missú. J. nat. Hist., $6: 229-241$.

Hamilton S.K. \& Lewis Jr. W.M. 1987. - Causes of seasonality in the chemistry of a lake on the Ornoco River floodplain, Venezuela. Limnol. Oceanogr., 32 : 1277-1290.

Hamilton S.K. \& Lewis Jr. W.M. 1989a. - Physical characteristics of the fringing floodplain of the Orinoco River, Venezuela. Interciencia, $15: 491-500$.

Hamilton S.K. \& Lewis Jr. W.M. 1990b. - Basin morphology in relation to chemical and ecological characteristics of lakes on the Orinoco River floodplain, Venezuela, Arch. Hydrobiol., $119: 393-425$.

Hamilton S.K., Sippel S.J., Lewis Jr. W.M. \& Saunders III J.F. 1990. - Zooplankton abundance and evidence for its reduction by macrophyte mats in two Orinoco floodplain lakes. J. Plankion Res., 12 ; 345-363.

Higler B. \& Statzner B. 1988 . - A simplified classification of freshwater bodies in the world. Verh. Internat. Verein. Limnol., 23 : 1495-1499.

Junk W.J. 1984. - Ecology of the varzea, floodplain of Amazonian whitewater rivers. In Sioli. H. (ed.), The Amazon: Limnology and Landscape Ecology of a Mighty Tropical River and its Basin. Monogr. Biol. no. 56. Junk. Dordrecht, 215-244.

Junk W.J., Bayley P.B. \& Sparks R.F. 1989. - The flood pulse concept in river floodplain systems. In Dodge, D.P. (ed.), Proceedings of the International Large River Symposium, Can. Spec. Publ. Fish. Aquat. Sci., $106: 110-127$.

Lewis Jr. W.M., Weibezahn F.H., Saunders III J.F. \& Hamilton S.k. 1990. - The Orinoco River as an ecological system. Interciencia, $15: 346-357$.

Neiff J.J., Patiño C.A. \& Martiarena N.R. - In press. Atenuación del escurrimiento fluvial por la vegetación del Bajo Paraguay durante las inundaciones. Seminario Internacional Hidrológico de Grandes Llanuras.

Paggi J.C. \& José de Paggi S. 1990. - Zooplankton of the lotic and lentic environments of the Middle Parana River. Acta Limnol. Brasil, 3 : 685-719. 
Rey J. \& Vásquez E. 1986. - Cladocères de quelques corps d'eaux du bassin moyen de l'Orénoque (Vénézuela). Annts Limnol., $22: 137.168$.

Robertson B.A. \& Hardy E.R. 1984. - Zooplankton of Amazonian lakes and rivers. In Sioli, H. (ed.), The Amazon : Limnology and Landscape Ecology of a Mighty rropical River and its Basin. Monogr. Biol. no. 56, Junk, Dordrecht, 337-352.

Saunders III J.F. \& Lewis Jr. W.M. 1989. - Zooplankton abundance in the lower Orinoco River, Venezuela. Limnol. Oceanogr., $34: 397-409$.

Shiel R.J. 1986. - Zooplankton of the Murray-Darling system. In Davies, B.R. \& Walker K.F. (eds.), The Ecology of River systems. Monogr. Biol. $n^{\circ}$ 60, Junk, Dordrecht, 661-677.

Tundisi J.C., Forsberg B.R., Devol A.H., Zaret T.M., Tundisi T.M., Dos Santos A., Rjbeiro J.S. \& Hardy E.R. 1984, Mixing patterns in Amazon lakes. Hydrobiologia, $108: 3-15$.

Twombly S. \& Lewis Jr. W.M. 1987. - Zooplankton abundance and species composition in Laguna La Orsinera, a Venezuelan floodplain lake. Arch. Hydrobiol. Suppl., $79: 87-107$.

Twombly S. \& Lewis Jr. W.M. 1989. - Factors regulating cladoceran dynamics in a Venezuelan floodplain lake. I. Plankton Res., 11 : 317-333.
Vásquez E. 1988. - Morfometria de un conjunto de lagunas dc inundación del Bajo Orinoco, Venezuela. Pantepuy, 4 : 31-37.

Vásquez E. 1989, - Caracteristicas morfométricas de algunas lagunas de la planicie aluvial bel bajo rio Orinoco, Venezuela. Mem. Soc. Cienc. Nat. La Salle, 49-50:309-227.

Vasquez E. 1993. - Temperature and dissoved oxygen in lakes of the Lower Orinoco River floodplain, Venezuela. Rev. Hydrobiol. trop., 25 (1) : (in press).

Vásquez E. \& Rey J. 1989. - A longitudinal study of zooplankton along the lower Orinoco River ans its Delta (Venezuela). Annls Limnol, 25 : 107-120.

Vásquez E. \& Sánchez L. 1984. - Variación estacional del plancton en dos sectores del rió Orinoco y una laguna de inundación adyacente. Mem. Soc. Cienc. Nat. La Salle, 44 (121) : 11-31.

Welcomme R, 1985, - River fisheries. FAO Fish. Tech. Pap., (262) : $330 \mathrm{p}$.

Wetzel R.G. 1990. - Land-water interfaces : metabolic and limnological regulators. Verh. Internat Verein. Limnol., 24 : 6-24. 